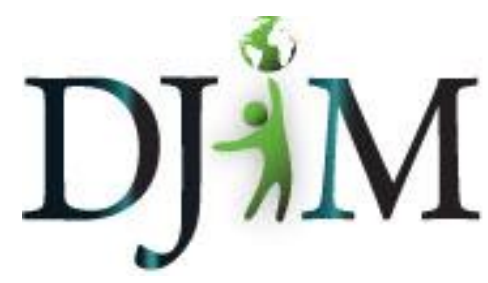

Volume 5 - Spring 2009

djim.management.dal.ca

\title{
A Different Kind of Memory: Examining the Effect of Technology through the Ages
}

\section{Robin Parker}

\begin{abstract}
The effect of new technologies on human memory is a subject of interest for educators, technology developers, and information specialists. However, such a preoccupation is not new to contemporary society. The current situation is examined by reflecting on the historical context of developments in technology and considering different perspectives on the significance of memory on personal and collective levels. This paper provides an overview of the contemporary understanding of how human memory functions as well as a discussion of the role of various types of memory in society.
\end{abstract}

\begin{abstract}
About the Author: Robin Parker is a first-year student in the Masters of Library and Information Studies at Dalhousie University. Her diverse interests are reflected in the dual fields of study from her undergraduate degree in general sciences and history. She hopes to continue pursuing her interests in the human and historical aspects of medicine through a career in health sciences librarianship. This paper was originally researched and written for INFO 5500, Information in Society course at the School of Information Management.
\end{abstract}

\section{Context of Question}

Reflecting on the difference between my use of memory and that of my grandparents generated the question of how the relationship between contemporary society and memory has changed. On the surface this appears to be a generational contrast. Despite their advanced ages, they are currently eighty-seven years old, the clarity of the memories they can retrieve of experiences that occurred forty, sixty, or even eighty years ago is startling. On the other hand, I have difficulty recreating memories from my childhood twenty years ago with comparable detail. Often memories I recall are only triggered by certain photographs that provide images to which I can attach other associations. While this anecdotal example of 
differences in recall abilities is subjectively based on personal experience, it suggests a topic worthy of further investigation.

These examples of episodic memory, defined as memories of autobiographical events previously experienced, are only one part of the impressive nature of my grandparents' memories (Block, 2002). My grandfather, in particular, can also recall specific information learned in a geology course taken in his junior year of university, over sixty years ago. Thus, his semantic memory, which is described as de-contextualized knowledge of the world, is also remarkably robust (Block, 2002).

The contrast in memory abilities between my grandparents and me is particularly notable given the generalization regarding memory decline in older individuals. Indeed, such a conception of memory lapses and decay in older populations is more than cultural bias; considerable amounts of social and medical resources and research focus on issues around memory loss associated with age (Rogers \& Fisk, 2006). To recognize that my grandparents are fortunate examples of good mental health partially explains the impressive nature of their memories. However, the markedly different approach to training their brains over the course of their lifetimes also must contribute to observable memory disparities. Changes in pedagogical techniques over the past century de-emphasizing learning by memorization have resulted in a decrease of mnemonic devices and related methods.

There are also inevitably other social factors that affect memory use and function in humans. One obvious difference between the world that my grandparents grew up in and our contemporary environment is the current proliferation of digital information and communication technologies. Suggestions that the responsibility for the lack of memory abilities of younger generations lies in the speed with which modern technology transfers information seems simplistic (Briscoe, 2000). However, the factors around the rapid pace of change surely contribute to evolving use of human memory.

\section{Introduction}

While the use of memory allows humans to engage in complex activities and intellectual endeavours, the limitations of human memory capacity create challenges that have been addressed in various ways throughout time. Whether through techniques to improve memory recall or technologies to supplement human memory capacity, humans have historically innovated new methods to retrieve information from past occurrences or observations. The various approaches to improve retrieval are influenced by contemporary understandings and appreciations of memory itself. Likewise, the functioning of human and supplemental memory capacities have shifted in response to evolving forms of use.

Issues around memory cannot be understood from a single perspective. In this paper I will examine the historical and modern understandings of memory and its role. By looking both 
from the perspective of individual memory processes and that of collective memory patterns, I will give an overview of the significance of memory. I will shed light on the social aspects of our changing relationship with memory through an examination of how technology has affected both our understanding of memory and the way that memory functions.

\section{Memory in Society}

Memory contributes to the most basic mode of information transfer in human experience. The link between memory and language as the root of communication goes back to the tradition of telling stories to pass information between individuals (Billington, 2001). Throughout human history, memory, whether individual or collective, stored internally or externally, has shaped identity and created continuity through time.

As pointed out by Susanna Radstone (2000), the level of interest in memory as a subject of cultural fascination, as well as academic and scientific study, has varied throughout history. The resulting amount of interest reflects the value placed on various forms of memory. However, due to memory's connection with the creation of knowledge and identity, there has always been some level of concern with memory or its lack. Especially in modern research, the failings of memory motivate the most intense investigation. Thus, memory loss due to disease, injury, or age is the subject of scrutiny by psychologists and neuroscientists (Samuel, 1999). In fact, the focus on memory issues and other cognitive problems resulted in the 1990s being declared the Decade of the Brain by UNESCO (Samuel, 1999).

\section{Descriptions of Personal Memory}

The complexity of the various definitions of memory and the ways that the different conceptualizations relate to humans and society challenges simplistic descriptions. First, there is the most basic definition of memory which applies to everything from individual to collective to technological forms of memory. This understanding describes memory as the capacity to store information and also the ability to retrieve that information (Memory, 2006). More detailed definitions impart the nuances of memory by revealing the diverse contexts of the word. Such definitions describe memory as a process, an impression or image stored in the brain, and the actual storage space for information (Memory, 1997; Memory, 2000; Memory, 2001).

The challenges faced in understanding the way individual human memory works have baffled people for as long as the subject of memory has fascinated them. Classical conceptions of memory located the corporeal aspects variously in the heart or blood (Samuel, 1999). In addition, personal memory was often divided into two categories from a classical point of view. The first is natural memory with which all individuals are born. The second is artificial memory that can be developed through its use and exercise in connection to learning (Yates, 1966).

Developments in neuroscience and psychology in the last century have gone a long way towards increasing understanding of the processes involved in human memory. By the time 
the field of psychology took precedence late in the nineteenth century, the brain was understood to hold the locus of memory, but the mechanisms were still very much unknown. Any understanding of the function and actions of memory achieved by drawing comparisons with mechanical processes invariably fell short. Thus, as neuroscientists developed theories of how memory works, more recent interpretations were also rejected. Namely, the idea of the mind acting as an information processor, in which memory plays the role of data storage, was a concept introduced in the 1950s, but is considered to be outdated by today's cognitive scientists (Johnson-Laird, 1988).

Our modern conception of human memory has developed a great deal through research conducted in the fields of psychology, neuroscience, and cognitive science. Individual memory is now understood to be made up of various components that are differentiated based on their functions. Most generally, the parts of memory are sensory memory, short-term memory (STM), and long-term memory (LTM) (Samuel, 1999). Each of these types of memory can be broken down into subcategories which operate in different ways and serve different cognitive functions. For example, LTM is comprised of procedural memory governing motor skills, conditioning, and perceptual learning as well as the semantic and episodic types of memory that were previously described (Magnussen, Endestad, Koriat, \& Helstrup, 2007; Samuel, 1999). Sensory memory consists of the brief preservation of sensory organ stimulus to allow for cognitive processing, while STM involves the retention of a small amount of information for a short span of time (Samuel, 1999).

Physical or procedural memory, as a subset of LTM, is the basis for many skills and habits used in daily life. Neuroscientists and cognitive scientists recognize the permanent aspect of this type of corporeal memory system, as compared to the somewhat more malleable characteristics of STM and language-based (known as declarative) types of LTM (Magnussen et al., 2007; Samuel, 1999). The complex interplay between all the parts that comprise human memory has made deciphering their various aspects and determining their roles that much more challenging for researchers.

Memory is central to learning and daily activities and as a result, it is often seen as being crucial to one's concept of self. The significance of individual and collective memory in creating identity serves as the basis for much philosophical debate and entire books have been dedicated to the topic (Blunstein, 2008). Certainly, without the ability to retrieve memories, it is impossible to achieve a sense of context in which to situate oneself. One must have a past concept of the self in order to direct activities in the present (Magnussen et al., 2007).

It is the ability to provide context that distinguishes human memory; the same fallibility of human memory represents its uniqueness. The connections that are made in human memory to recall a piece of information are complex and difficult to replicate. Neuroscientist JeanGabriel Ganascia describes the working of human memory as "much more than simple information processing ... and [it is an] inordinately rich web of self-reflexive, interweaving 
recollection that no computer has ever come close to imitating" (as cited in Briscoe, 2000, p. 45). Human memory and technological memory capacity will be further elaborated to emphasize the divergent roles of the two phenomena.

\section{Historical Perspectives on Memory and Knowledge}

Definitions of memory frequently include reference to its role in learning. As Samuel (1999) states, "learning and memory were for decades semantic twins" (p. 2). The connection continues from learning on to knowledge and wisdom. In classical times, memory was considered "the mother of all wisdom ... [and] the treasury and guardian of all things" (Boornstin, 1983, p. 483). Quite literally, for the Greeks, "Mnemosyne, the goddess of memory, was also the goddess of wisdom and the mother of the muses" (Samuel, 1994, as cited in Radstone, 2000, p. 2). Thus, for classical scholars, any degradation of memory would result in a decrease in the ability to learn and retain knowledge.

The historical significance of the use of human memory in connection to knowledge is described in great detail in The Art of Memory by Frances Yates (1966). In this pivotal work, the methods of ancient and medieval memory techniques are elaborated and put into the context of their historical development and significance in society. For the ancient art of memory, rhetoric played a crucial role as memorization was the only way to learn orations in non-literate societies. The background provided in Yates' book illustrates in great detail how artificial memory played an important role in the creation and transfer of knowledge throughout human history.

The understanding of artificial memory as an ability to be refined with years of learning and practice was most valued in non-literate cultures. As Ong (1982) points out, mnemonic exercises and formulations preserved and transferred knowledge without a written record. However, the use of memory as an art is not isolated solely to oral societies. Due to the rarity of manuscripts, memory still played a very important part in the transfer and use of knowledge in the Western world until the advent of printing in the fifteenth century (Boornstin, 1983). Indeed, even after the proliferation of books, for at least another two hundred years the expense of books and the restricted ability to read them privileged memory over the written transfer of information for most people. While books could transfer information much more quickly and accurately, memorizing information imparted by word of mouth was still the only effective means of knowledge transmission for the poor and uneducated majority (Boornstin, 1983).

Thus, many hundreds of years passed before the role of individual human memory in education and the transmission of knowledge became less critical. Even as late as the first half of the twentieth century students experienced the pedagogical emphasis on mnemonic techniques. Individuals in school in the early 1900 s would have learned through memorization much more than was common later in the century (Muske-Dukes, 2002). However, memory is 
still understood to play an important role in learning and is not overlooked by instructional methods (Parker, 1993; Weiss, 2000). Contemporary courses requiring the memorization of poetry, for example, enhance the comprehension of the texts while mimicking the teaching methods of the previous century (Muske-Dukes, 2002).

\section{Collective Memory}

On the collective level, the functioning of memory is equally complex. The means of preserving societal memory and the value placed on such memory changes greatly depending on the context. Blunstein (2008) describes the political role of collective memory as being "used and manipulated to strengthen and serve the authority of the ruling powers or to challenge that authority" (p.187). Certainly what gets remembered and preserved through memory institutions and cultural artefacts varies depending on where the power lies in a given society.

Similar to the role of personal memory in creating one's sense of self, collective memory is crucial to the formation of a cultural identity. In an essay comparing the portrayal of memory and technology in two popular films, memory is described as "a crucial force in the creation of culture and cultural images; in what we call a cultural self" (Blackmore, 2004, p. 17). The significance of the modes of memory preservation on a societal level is therefore of considerable importance. Such means of creating and maintaining a cultural sense of self are not always equally valued.

Much like the varying degrees of interest in individual memory, the regard with which collective memory institutions and artefacts are held depends on the worth of the cultural conceptualization. Thus, libraries, museums, and archives themselves, as well as their contents, are evidence of the worth assigned to traditions and customs. Another factor is the extent to which a given society desires to project a sense of history and continuity into the future. Pessach (2008) describes one of the determining aspects of the nature of memory institutions: "[t]he first dimension is intergenerational and it refers to the importance of providing future generations with as many landscapes of culture and history as possible" ( $p$. 74). Just as earlier societies told stories to convey messages to future generations, collective memory institutions reflect forward a sense of the past and present (Donald, 1991).

\section{Historical Intersections of Technology and Memory}

Regardless of the type of memory being considered, it is closely connected to technology in its assorted formats. Ever since Western societies embraced literacy and departed from a culture of orality, the externalization of human memory has evolved. Plato expressed concern about the "inhuman" nature of writing and in Phaedrus, statedthat the written word has a destructive effect on human memory (Ong, 1982). The contemporary interpretations of the relationship between memory and digital technology emphasize the possibility that the former will replace the latter. But for centuries, humans have been striving to improve the efficiency of the 
externalization of memory in order to free attention for other purposes. Indeed, it can be argued that much of the progress of Western civilizations, from philosophy to electricity, depends on abstract thoughts extrapolating from previously recorded concepts.

Over the course of the centuries that separate the oral past from the digital present, civilizations rose and fell while countless technological and scientific developments took place. Today, acceptance of the interaction between technology and human accomplishment is such that the two are inextricably intertwined. Donald (2004) states: "much of what we call 'higher' intelligence is a product of marrying the raw intellectual power of the human brain to an appropriate technology. Literacy-related skills are the most obvious examples of this" (p. 379). The Western world as it now stands would not be possible without the externalization of memory in the form of writing accompanied by drastic social changes over time (Donald, 2001).

Technology has been used not only to preserve, but also to propagate information that was once the territory of memory. Boornstin (1983) describes the enormous impact on the Western world due to the introduction of the printing press. With the proliferation of printed books, ideas spread much more quickly and abstractions resulting from those ideas were easier to pass on and develop amongst the learned. As the volume of information grew, improvements in the structure of the printed format, such as continuous pagination, tables of contents, and indexes allowed easier reference to the information contained within books (Boornstin, 1983). All of these developments simultaneously eased the burden on human memory and increased the available amount of information the mind could access and potentially use.

\section{Historical Role of Technology as Memory's Aid}

Directly following the Second World War, Vannevar Bush discussed the importance of preserving an external record of the processes and elements involved in scientific research. He emphasized the crucial role of developing better methods of creating and protecting documentation in order to free the mind of the scientist for more creative or innovative thought (Bush, 1999). To this end, Bush proposed a memex device which would consist of "a microfilm machine that stored and retrieved vast amounts of information" (Yeo, 2007, para. 1).

However, Bush was not the only, or even the first, thinker to contemplate externalization of memory as the means for advancement of humankind. As early as the seventeenth century, philosophers Robert Hooke and John Locke were each separately focused on the excess of information available (Yeo, 2007). They "were concerned about information overload and the desirability of efficient methods of storing and finding data" (Yeo, 2007, para. 11). In his essay, Yeo (2007) situates the three men as historical examples of the trend towards increasing externalization of memory as a means for scientific and philosophical progress. 
Bush was prescient in his vision of ways that technology could develop to replace the more mundane activities of human memory. In fact, he was remarkably accurate in his predictions of the future role of information technologies if not the actual form of the technologies themselves. The dream he held regarding a storage device smaller than a matchbox capable of holding the information contained in the Encyclopaedia Britannica has indeed come to fruition in the form of flash drives of enormous memory capacities.

\section{Concerns about Technology}

As described by Ong (1982), the hesitations that Plato expressed over writing have echoed through time with the introduction of the printing press and most recently with the prevalence of computers and digital technologies. As with many trends in human interaction with information, our modern concerns are not the first expression of fear around change and the power of information (Rosenberg, 2003). In the case of memory, the unknowns lie in the potentially positive or negative effects of externalization of memory from the human mind. In addition, the specific role of memory in the creation of the human self continues to defy understanding. The combination of these uncertainties results in fear surrounding the changes in the contemporary use of human memory.

However, in many ways, the artificial memory described by Yates filled the same function now assigned to external versions of memory. Both artificial memory and external memory in the form of various technologies serve to enhance and support the functioning of natural memory (Yeo, 2007).

While popular literature often makes comparisons between the nature of human memory and computer memory, the similarities are only surface deep (Harris, 2007). Both phenomena are responsible (at least in part) for the retention of information, but the processes and structures involved are not particularly comparable. Whereas computer memory is characterized as being like a series of pigeonholes into which information is placed, human memory has no such comparable structure (Johnson-Laird, 1988; Loftus \& Calvin, 2001).

To compensate for the lack of a physiological structure to tuck information into, early orators used a metaphoric construction of physical space as a repository for memories. Yates (1966) and others describe the architectural method of memorization used in ancient times (Blackmore, 2004; Ong, 1982). According to this technique, the individual would assign certain sets of information, often in the form of words, phrases, concepts or pictures, to the parts of an imagined building. To retrieve the memory or speech, the person would then walk mentally through the building, recognizing the desired pieces of information in their appropriate locations.

In the contemporary world, the prolific use of digital technologies as "outboard brains" to supplement everyday memory assists all sorts of daily activities (Thompson, 2007). Personal 
digital devices serve as crutches to human memory by holding seemingly irrelevant information ready for retrieval. The important question to ask involves where the line is between the use of technology to assist human activity and that to replace human intelligence. If Western society is relying on technology to do the thinking, then the ability to choose what will be remembered will be lost.

The significant effect of technological developments on the use and function of human memory is not exaggerated. Countless opinion pieces bemoan the loss of effective human memory as a result of dependence on personal memory devices (Briscoe, 2000; Carr, 2008; Johnson, 2000; Walshe, 2008). The influence of daily habit on brain structure is substantiated by recent neuroscience and psychology research indicating the surprising degree of plasticity of the human brain. Evidence of the benefits of training older adults' memories supports the concept that "induced plasticity seems to be possible even in advanced old age" (Buschkuehl et al., 2008, p. 751). It turns out that the way that we use our minds has a physical effect of rewiring the organization of the brain. This can have very real implications for human memory, giving truth to the saying "use it or lose it."

That said, the state of understanding regarding the biological processes involved in the production of memories and the physiological structures responsible for their retention and recall is still imperfect. So while current empirical evidence indicates a decrease in functional memory and the ability to retrieve information without the aid of digital devices, the trade-off in mental capacity is not clear (Pinto, 2007). Arguments have been made that the result is the ability to devote more energy to creativity and innovation (Bush, 1999). However, the working capacity of human memory has never been successfully calculated. Best guesses are so large that arguments of running out of space seem absurd (Samuel, 1999). More relevant concerns involve attention and the limited speed at which new information can be integrated into memory formats (Samuel, 1999). In addition, without the assurance that the occupation of brain space or energy works in a cumulative sense, it is not certain that there is a direct correlation between less use of memory and higher use of intellect. That the opposite may be true is of concern, particularly regarding the future role of human intelligence in managing the information externalized out of human memory (Donald, 1991).

Another consideration regarding the externalization of memory is how easily specific information can be retrieved from the vast quantity of information contained within a digital storage device or a human mind. With the modern sense of information overload, extraction of pertinent information from the mass at large has become a growing concern. The current inability to effectively retrieve information, whether due to the degradation of human memory or as a result of the sheer volume of information available is an object of concern and research (Huyssen, 2000). Computer scientists strive to create devices designed to assist individuals in remembering where information is stored and cue the process to recover that information (Elsweller, Ruthven, \& Jones, 2007). 
Another layer of the interrelationship between memory and technology is due to the role of computer technology in neuroscience research. The contemporary understanding of the processes and structures involved in memory are a result of the development of fast computers that are capable of dealing with and modelling the complicated interactions that take place in the brain (Samuel, 1999). Thus, it is thanks to advancements made in computer science that it is possible to come closer to a conceptualization of the functioning of the brain in general and memory specifically.

\section{Current Perceptions of Technology and Memory}

Research conducted within the last twenty years helps substantiate the theory that externalization of memory through use of symbolic notation affects the functioning of human memory. In a study conducted by Michelle Eskritt, Kang Lee, and Merlin Donald (2001), their conclusions supported the idea that writing affects the work of memory, but they emphasized the nuanced results of their research. Their results "imply that the influence of external symbols on memory is more complex than originally supposed. [...] Thus, the introduction of external symbols leads to an adaptive use of our memory resources" (Eskritt, Lee \& Donald, 2001 , p. 48). As previously mentioned, neuroscientists are increasingly using theories regarding the plasticity of the brain to understand the changes in the functioning of memory due to technology use.

As Bush (1999) postulated, external memory technologies of increasing capacity and efficiency play important roles in freeing the human mind to engage in occupations for which it is well suited. Creativity, imagination, and innovation are results of a flexibility of mind that is permitted though the external storage and sorting of some of the information produced by thought processes. However, the effectiveness of externalized memory is contingent on how well that information can be retrieved at will; in other words, how efficiently cataloguing and searching is performed on the information, as discussed earlier.

\section{Collective Memory and Technology}

The methods and functions of collective memory have also been greatly affected by modern digital technologies. As opposed to the traditional institutions of cultural memory preservation that have developed in the West in the form of libraries, museums, and archives, current techniques of remembering are often ensconced in the networked universe of the Internet. With the ever-increasing efforts to digitize the records of the formerly unchallenged memory institutions, the responsibility for preservation of cultural memory is shifting (Pessarch, 2008). Merlin Donald (2001) describes the shift in paradigms: "in modern society control is no longer held by such institutions, and there is no way back. As it has grown with accelerating and frightening inevitability, the technology of memory has broken the system wide open" (para. 35). 
Another factor concerns the longevity of the storage method used. This is a particularly significant issue when considering the storage of cultural memory artefacts over a long period of time in order to preserve them for future generations. Thus, the function of collective memory institutions to retain and safeguard the knowledge of society is achieved only so far as the information is retrievable for future generations.

Analog versions of cultural memory in the form of books, film, and audio recordings are subject to issues of preservation. In particular, paper-based records produced during the period of high-acid paper in the past century are in the process of disintegration (Billington, 2001). Yet the obvious answer of the digitalization of records also presents problems. Another risk that society is currently addressing with the digitization of cultural records concerns the obsolescence and decay of modern technology. In an essay addressing the role of media and politics in individual and cultural forgetting, Andreas Huyssen (2000) puts this issue in no uncertain terms: "the threat of oblivion emerges from the very technology to which we entrust the vast body of contemporary records and data, that most significant part of the cultural memory of our time" (p. 36). Without an effective method to protect against the problems inherent in the speed of technological change, Western society risks losing its memory of itself.

Nevertheless, forgetting is a crucial part of remembering. As early as John Locke (1632-1704), there was an understanding that in order to remember some things, human memory must forget many details (Yeo, 2007). In his imaginings of the potential capacities of external memory technologies, Bush (1999) waxes eloquent regarding the efforts of the future scientist: "[h]is excursions may be more enjoyable if he can re-acquire the privilege of forgetting the manifold things he does not need to have immediately at hand, with some assurance that he can find them again if they prove important" (p. 187-188). Huyssen (2000) also emphasizes the role of forgetting by invoking Freud, who had "already taught us that memory and forgetting are indissolubly linked to each other, that memory is but another form of forgetting, and that forgetting a form of hidden memory" (p. 27). These various examples of the interplay of forgetting and memory demonstrate the complexity into which technology enters.

\section{Into the Future of Technology and Human Memory}

While current debates rage on about the value and consequences of technological infringement on human memory, it is inevitable that technology will continue to change. Developments in information technology are apparent on an almost daily basis, as seen with the ever-expanding empire of Google. The question remains: what will the relationship between technology and human memory will look like in the not-so-distant future?

The innovations in technology that aid the ailing memories of older adults and victims of brain trauma have the potential to greatly improve quality of life (Rogers \& Fisk, 2006). Likewise, research aided by technology will continue to contribute to scientists' understanding of the mechanisms of the brain and memory itself, allowing further progress in treating failures and weaknesses in the functioning of human memory. 
Technology-heavy visions of the future often include versions of integrated computer and human cognitive processes to augment the frailty of human memory (Briscoe, 2000; Pedersen, 2008; Warwick, 2004). Such drastic alterations of the structure of the human mind and experience would lead to a future that is unrecognizable from the present perspective. But even if the future reality is not so extreme, the humans existing therein will have inevitably changed. We will have adapted to, and with, technology just as modern-day humans have evolved from our early predecessors who were unfamiliar with any type of externalized symbolic forms of memory.

Conversely, perhaps the continued expansion of external memory storage systems will render human memory more redundant and unnecessary. As the means of storing and retrieving information in external, networked, and often social formats grows increasingly sophisticated, the need for the human mind to perform those functions may become less relevant. Perhaps, as with the effect of writing, the human mind will diversify its activities to attain new levels not dreamed of as yet (Eskritt et al., 2001).

The complex ability of human memory to store information within a contextualized environment has not yet been met by technological developments. At the current rate at which RAM is increasing, the capacity of digital memory will soon exceed that of human memory, but the functions of human memory in connection to the whole of the brain are more challenging to match. It is possible that the complex interactions on which knowledge acquisition depends will lose the support of a smoothly functioning memory system as the result of inactivity (Thompson, 2007). As Donald (1991) describes elsewhere, the structure and function of human memory is continuously under revision:

Although we have known for some time that the human cortex is a remarkably malleable instrument, perhaps we have underestimated its flexibility, or to use the correct word, its plasticity. We are still changing; the final structure of human memory systems, if they ever reach a steady state, lies somewhere in the future. (para. 7)

On the collective memory side, questions of what the future holds are equally perplexing. While it seems inevitable that the storage of the majority of cultural records is occurring in the digital realm, issues regarding preservation and access remain unresolved. Networked storage systems as well as networked access to information could increase opportunities for more individuals to obtain socially-created information. However, concerns about the future of networked cultural memory institutions revolve around issues of privatization that could effectively restrict access to certain information (Pessach, 2008). Worries regarding the loss of structure also figure in the musings of those looking at the changing role and nature of memory institutions in society (Donald, 2001). 


\section{Conclusions}

Our use of tools to accomplish complex activities is one thing that makes us innately human. The technology we use to help us think is no different. The dynamic relationship between tools, embodied in technology, and the human mind will help determine the nature of the humans that we are in the process of creating. In the context of both individual memory and the collective forms of cultural memory, technology will continue to shape and be shaped by how we manage our information needs. The value that is placed on various types of information, in the form of memories, will determine how concerned we are with the ways that we store, retrieve, and manage that information. The role that human memory will play in that process is currently being altered, and will continue to change as time progresses along with human civilization.

The crucial responsibility of the human mind is in its ability to reflect on information and filter that data though the complex web of experience that shapes consciousness. Memory is indispensable to the process of integrating new information with already known facts (Alsop, 2005; Carr, 2008). In order to become more knowledgeable, one must be able to reflect on existing knowledge and amalgamate it with what one already knows. Despite this fact, the ability to recall everything one has ever learned is not necessary for an overall increase in knowledge.

Even my grandparents cannot remember all the details of their past or the lessons they have learned. But by constantly incorporating new information into their existing matrices of knowledge, they build on the rich history of their lives. I hope that by following the example they set and by judiciously using the assistance that externalized memory storage provides, I too will regale future generations with stories of my experiences.

Certainly, with the quantity of information that is available to me, technological devices will help me find and preserve a record of significant information for my needs. Whether that technology takes the form of a written record, a digital phone list, or an electronic database, the responsibility will still lie with my memory to determine when and which information is needed, and for what purpose. If I can do that, then hope is not lost for the memory of my generation and the generations of the future.

\section{References}

Alsop, J. (2005). Losing our minds. College \& Research Libraries News, 66(11), 790-791, 838. Retrieved from Academic Search Premier database.

Billington, J. (2001). Culture, memory and technology. Sewanee Review, 109(2), 218. Retrieved from Academic Search Premier database. 
Blackmore, T. (2004). High on technology - Low on memory: Cultural crisis in Dark City and The Matrix. Canadian Review of American Studies, 34(1), 13-54. Retrieved from Academic Search Premier database.

Block, M. (2002). Memory. Encyclopedia of social and cultural anthropology. London, United Kingdom: Routledge. Retrieved from CREDO.

Blunstein, S. (2008). The moral demands of memory. New York: Cambridge University Press.

Boornstin, D. J. (1983). The discoverers: A history of man's search to know his world and himself. New York: Vintage Books.

Briscoe, I. (2000, December). When computers chip away at our memories. UNESCO Courier, 53(12), 44-45. Retrieved from Academic Search Premier database.

Bush, V. (1999). As we may think. Library Computing, 18(3), 180-188. Retrieved from ABI/INFORM Global database.

Buschkuehl, M., Jaeggi, S., Hutchison, S., Perrig-Chiello, P., Däpp, C., Müller, M., et al. (2008, December). Impact of working memory training on memory performance in old-old adults. Psychology and Aging, 23(4), 743-753. doi:10.1037/a0014342

Carr, N. (2008, July/August) Is Google making us stupid? The Atlantic. Retrieved November 24, 2008, from http://www.theatlantic.com/doc/200807/google

Donald, M. (1991, December 26). An urgent matter of the mind. The Globe and Mail, p. A22. Retrieved from ProQuest Newsstand database.

Donald, M. (2001). Memory places: The revolutionary function of libraries. Queen's Quarterly, 108(4), 558-573. Retrieved from CBCA Complete database.

Donald, M. (2004). Is a picture really worth 1,000 words?[Review of the book: Computers, visualization, and history: How new technology will transform our understanding of the past]. History and Theory, 43, 379-385. doi: 10.1111/j.1468-2303.2004.00289.x

Elsweiler, D., Ruthven, I., \& Jones, C. (2007). Towards memory supporting personal information management tools. Journal of the American Society for Information Science \& Technology, 58(7), 924-946. doi:10.1002/asi.20570

Eskritt, M., Lee, K., \& Donald, M. (2001). The influence of symbolic literacy on memory: Testing Plato's hypothesis. Canadian Journal of Experimental Psychology, 55(1), 39-50. Retrieved from Canadian Periodicals database.

Harris, D. (2007, May 24). So, what was that memory technology again? Electronic Design, 55(11), 50-54. Retrieved from Academic Search Premier database. 
Huyssen, A. (2000). Present pasts: Media, politics, amnesia. Public Culture, 12(1), 21-38. Retrieved from International Political Science Abstracts database.

Johnson-Laird, P.N. (1988). The computer and the mind: An introduction to cognitive science. Cambridge, MA: Harvard University Press.

Loftus, E., \& Calvin, W. (2001). Memory's future: Will technology change the way human memory works? Psychology Today, 34(2), 55. Retrieved from CINAHL with Full Text database.

Magnussen, S., Endestad, T., Koriat, A., \& Helstrup, T. (2007).What do people believe about memory and how do they talk about memory? In S. Magnussen \& T. Helstrup (Eds.), Everyday Memory (pp. 5-25). New York: Psychology Press.

Memory. (1997). The Macquarie dictionary. South Yarra, VIC, Australia: The Macquarie Library Pty Ltd. Retrieved from CREDO.

Memory. (2000). The Penguin English dictionary. London, United Kingdom: Penguin. Retrieved from CREDO.

Memory. (2001). Chambers 21st century dictionary. London, United Kingdom: Chambers Harrap. Retrieved from CREDO.

Memory. (2006). Dictionary of Information and Library Management. London: A\&C Black. Retrieved from CREDO.

Muske-Dukes, C. (2002, December 29). A lost eloquence [Op-Ed]. New York Times, pp. 4-9. Retrieved from Banking Information Source database.

Ong, W. J. (1982). Orality and literacy: The technologizing of the word. New York: Methuen.

Parker, J. K. (1993). Lecturing and loving it: Applying the information-processing model. The Clearing House, 67(1), 8-11. Retrieved from Research Library database.

Pedersen, I. (2008). MyLifeBits, augmented memory, and a rhetoric of need. Continuum: Journal of Media \& Cultural Studies, 22(3), 375-384. doi:10.1080/10304310801919429

Pessach, G. (2008). [Networked] Memory institutions: Social remembering, privatization and its discontents. Cardozo Arts \& Entertainment Law Journal, 26(1), 71-149. Retrieved from Film \& Television Literature Index with Full Text database.

Pinto, J. (2007, December). Automation is a crutch. InTech, 54(12), 10. Retrieved from Research Library database.

Radstone, S. (2000) Working with memory: an introduction. In S. Radstone (Ed.), Memory and Methodology (pp. 1-21). New York: Berg. 
Rogers, W. A., \& Fisk, A. D. (2006). Cognitive support for elders through technology. Generations, 30(2), 38-43. Retrieved from Academic Search Premier database.

Rosenberg, D. (2003). Early modern information overload. Journal of the History of Ideas, 64(1), 1-9. Retrieved from Research Library database.

Samuel, D. (1999). Memory: How we use it, lose it and can improve it. New York: New York University Press.

Thompson, C. (2007, September 25). Your outboard brain knows it all. Wired, 15(10). Retrieved 25 November, 2008 from http://www.wired.com/techbiz/people/magazine/1510/st_thompsonhttp://www.wired.com/techbiz/people/magazine/15-10/st_thompson

Walshe, E. (2008, September 11). Who needs a good memory when there's Google? The Christian Science Monitor, 9. Retrieved from ProQuest Newsstand database.

Warwick, K. (2004, June). Headline from the future. Popular Science, 264(6), 54. Retrieved from Academic Search Premier database.

Weiss, R. P. (2000). Memory and learning. Training \& Development, 54(10), 46-50. Retrieved from ABI/INFORM Global database.

Yates, F. A. (1966). The art of memory. London: Routledge and Kegan Paul.

Yeo, R. (2007). Before memex: Robert Hooke, John Locke, and Vannevar Bush on external memory. Science in Context, 20(1), 21-47. Retrieved from Historical Abstracts database. 